\title{
Resveratrol Induces Glioma Cell Apoptosis through Activation of Tristetraprolin
}

\author{
Jinhyun Ryu', Nal Ae Yoon', Hyemin Seong ${ }^{1}$, Joo Yeon Jeong ${ }^{1}$, Seokmin Kang ${ }^{1}$, Nammi Park², Jungil \\ Choi', Dong Hoon Lee', Gu Seob Roh', Hyun Joon Kim', Gyeong Jae Cho', Wan Sung Choi', Jae-Yong \\ Park, Jeong Woo Park, , and Sang Soo Kang ${ }^{1, *}$
}

\begin{abstract}
Tristetraprolin (TTP) is an AU-rich elements (AREs)binding protein, which regulates the decay of AREscontaining mRNAs such as proto-oncogenes, antiapoptotic genes and immune regulatory genes. Despite the low expression of TTP in various human cancers, the mechanism involving suppressed expression of TTP is not fully understood. Here, we demonstrate that Resveratrol (3,5,4' -trihydroxystilbene, Res), a naturally occurring compound, induces glioma cell apoptosis through activation of tristetraprolin (TTP). Res increased TTP expression in U87MG human glioma cells. Res-induced TTP destabilized the urokinase plasminogen activator and urokinase plasminogen activator receptor mRNAs by binding to the ARE regions containing the 3 untranslated regions of their mRNAs. Furthermore, TTP induced by Res suppressed cell growth and induced apoptosis in the human glioma cells. Because of its regulation of TTP expression, these findings suggest that the bioactive dietary compound Res can be used as a novel anti-cancer agent for the treatment of human malignant gliomas.
\end{abstract}

\section{INTRODUCTION}

Malignant gliomas are the most common primary tumors arising within the brain (Nakada et al., 2007). Although surgery and subsequent chemotherapy are the usual treatments for gliomas, most malignant gliomas are strongly resistant to apoptosis in-

\footnotetext{
${ }^{1}$ Department of Anatomy and Convergence Medical Science, Institute of Health Science, School of Medicine, ${ }^{2}$ Department of Physiology, Institute of Health Sciences, School of Medicine, Gyeongsang National University, Jinju 52727, Korea, ${ }^{3}$ Gyeongnam Department of Environmental Toxicology and Chemistry, Korea Institute of Toxicology (KIT), Jinju 660-844, Korea, ${ }^{4}$ School of Biosystem and Biomedical Science, College of Health Science, Korea University, Seoul 02841, Korea, ${ }^{5}$ Department of Biological Sciences, University of Ulsan, Ulsan 680-749, Korea

*Correspondence: kangss@gnu.ac.kr (SSK), jwpark@ulasan.ac.kr (JWP).
}

Received 14 July, 2015; revised 3 September, 2015; accepted 7 September, 2015; published online 4 November. 2015

Keywords: apoptosis, glioma, resveratrol, TTP, uPA, uPAR duced by chemotherapeutic agents. Because several mechanisms, including the blood-brain barrier (BBB), restrict and control the sufficient delivery of chemotherapeutic agents, the prognosis of patients with malignant gliomas is very poor (Stupp et al., 2007; Villano et al., 2009). To treat malignant gliomas using chemotherapy, it is therefore necessary to use agents that readily pass through the BBB.

Resveratrol (3,5,4'-trihydroxystilbene, Res), a polyphenol compound found in diverse sources such as grape skin, red wine, various berries, peanuts, and other dietary products, is a potent anti-oxidant (Cucciolla et al., 2007; Dorai and Aggarwal, 2004; Fremont, 2000). Res has been reported to have antiproliferative properties against several cancers such as breast, liver, pancreas, and colon cancers (Cucciolla et al., 2007). Res acts as a chemopreventive and anti-tumor agent, in vivo, by inhibiting a variety of carcinogenic processes at diverse stages (Jang et al., 1997). Res also suppresses cell cycle progression (Ahmad et al., 2001), migration, and invasion (Weng et al., 2010), and induces apoptosis (Tinhofer et al., 2001; Trincheri et al., 2007). Previous studies have reported that Res inhibited glioma progression (Jiang et al., 2005; Ryu et al., 2011), specifically by passing through the BBB (Wang et al., 2002). In some clinical tests, orally administered Res at high doses to healthy volunteers was safe and well-tolerated, supporting its potential utilization as an anti-cancer agent (Boocock et al., 2007; Cottart et al., 2010).

Tristetraprolin (TTP) is an AU-rich elements (AREs)-binding protein that regulates the decay of ARE-containing mRNAs, such as proto-oncogenes, anti-apoptotic genes, and immune regulatory genes (Baou et al., 2009). TTP also regulates the decay of urokinase plasminogen activator (UPA) and urokinase plasminogen activator receptor (UPAR) transcripts (Ryu et al., 2015), suggesting a potential role for TTP in invasion and tumor growth. A previous study reported that TTP expression was suppressed in human cancers, such as lung, breast, and cervix, and that the decline of functional TTP mediated diverse tumorigenic phenotypes (Brennan et al., 2009). However, the mechanism of TTP deficiency in cancer is not fully understood, and the development of novel agents to increase endogenous TTP expression is currently a high priority.

Here, we demonstrate that Res induces glioma cell apoptosis through activation of TTP. The expression of Res-induced TTP destabilized UPA and UPAR mRNAs by binding to the ARE 
regions containing $3^{\prime}$ untranslated regions ( $3^{\prime}$ UTR) of their mRNAs. Furthermore, increased expression of TTP by Res suppressed cell growth and induced apoptosis in human glioma cells. Together, these results suggest that Res can be used as an anti-cancer agent to treat human malignant gliomas by the regulation of the endogenous expression of TTP.

\section{MATERIALS AND METHODS}

\section{Cells and treatment}

U87MG human glioma cells and normal human fibroblast (NHFB) cells (ATCC, USA) were maintained in the high glucose Dulbecco's minimal essential medium (DMEM; Gibco, USA) supplemented with $10 \%$ fetal bovine serum (FBS; Gibco), 100 $\mathrm{U} / \mathrm{ml}$ penicillin, and $100 \mu \mathrm{g} / \mathrm{ml}$ streptomycin (P/S; Gibco) in $5 \%$ $\mathrm{CO}_{2}$ at $37^{\circ} \mathrm{C}$. For some studies, U87MG and NHFB cells were cultured in DMEM without FBS in the presence of designated concentrations of $\operatorname{Res}(5-200 \mu \mathrm{M})$ (Tocris, USA).

\section{Reverse transcription (RT)-PCR}

Total RNA was extracted using TRIzol reagent (Invitrogen, USA) following the manufacturer's instructions. Total RNA (1 $\mu \mathrm{g}$ ) was used for cDNA synthesis using an iCycler thermocycler (Bio-Rad Laboratories, USA). RT-PCR was performed using rTaq polymerase (Elpis Biotech, Korea). Primers were synthesized based on the reported human CDNA sequences for UPA, UPAR, and hGAPDH in the NCBI data bank. Sequences of the primers used for PCR were as follows; TTP: AGGCCAATCGCCACCCCAAA, GTGCCAGGGGCAGCAGA GAA; UPA: ACTTCTCCAACATTCACTGG, ATTCTTCTGG AGGAGAGGAG; UPAR: TGCCCGGGCTCCAATGGTTT, ATT CTTCTGGAGGAGAGGAG; hGAPDH:AGCTGAACGGGAAG CTCACT, TGCTGTAGCCAAATTCGTTG (Bioneer Corporation, Korea).

\section{Quantitative real-time PCR (Q-PCR)}

For RNA kinetic analysis, UPA and UPAR mRNAs were assessed in the presence of actinomycin D (Sigma-Aldrich, USA) by quantitative real-time PCR (Q-PCR). Q-PCR was performed using EvaGreen qPCR Mastermix (Applied Biological Materials Inc., Canada) in a Light Cycler 480 II (Roche Applied Science, USA). The results were analyzed by the melting curve analysis and agarose gel electrophoresis. PCR primer pairs were as follows: quPA: CCCTGCCTGCCCTGGAACTC, AGCGGCTT TAGGCCCACCTG; quPAR: CATTGACTGCCGAGGCCCCA, TGCTGAAGGCGTCACCCAGG; qhGAPDH: GCACCCCTG GCCAAGGTCAT, ACGCCACAGTTTCCCGGAGG (Bioneer Corporation).

\section{Western blotting}

Equivalent amounts of total protein $(20-30 \mu \mathrm{g})$ were separated by SDS-PAGE on $8-12 \%$ polyacrylamide gels and then transferred to nitrocellulose membranes (Whatman $\mathrm{GmbH}$, Germany) using a semi-dry transfer apparatus (Bio-Rad Laboratories) submerged in transfer buffer (25 mM Tris, $192 \mathrm{mM}$ glycine, $20 \%$ methanol, $\mathrm{pH} 8.3$ ). The membrane was blocked with $5 \%$ skim milk in $0.1 \%$ Tween-20/Tris-buffered saline (TTBS), and then incubated with the appropriate dilutions of primary and secondary antibodies. Antibodies to the following proteins were used: TTP, $\alpha$-tubulin, and $\beta$-actin (Sigma-Aldrich), and uPA and UPAR (Epitomics, USA). Immunoreactivity was detected by chemiluminescence (ECL; Advansta, USA) using LAS 4000 (Fujifilm, Japan).
Plasmids, transfection, and luciferase assay U87MG cells were transfected with the TTP-expressing pcDNA6/V5-HisA vector (pcDNA6/N5-TTP) (Invitrogen) (Lee et al., 2010) using the TurboFect ${ }^{\mathrm{TM}}$ in vitro transfection reagent (Fermentas, USA) for the overexpression of TTP.

For luciferase assays, U87MG cells were co-transfected with the designated construct, such as psiCHECK-Frag-uPA or UPAR 3' UTR, and pcDNA6/N5-TTP in vitro using the TurboFect ${ }^{\mathrm{TM}}$ transfection reagent (Fermentas); the U87MG cells were then treated with Res. Cells were lysed with lysis buffer (Promega, USA), mixed with the luciferase assay reagent (Promega), and the chemiluminescent signal was measured by the Infinite M200 Pro (Tecan, Switzerland). Firefly luciferase was normalized to Renilla luciferase in each sample. All reported luciferase assays represented at least three independent experiments, each consisting of three wells per transfection.

\section{Electrophoretic mobility shift assay (EMSA)}

The biotinylated RNA probes for the wild-type uPA-ARE-WT, ACUCCUGUACACUGAAUAGCAUAUUUCACUAUUUUUAU UUAUAUUUUUGUAAUUUUAAA; and UPAR-ARE-WT, GUUG UUGUUAUUAAUUAAUAUUCAU-AUUAUUUAUUUUAUACU CAUAAAGAUUUUGUACC were synthesized by Samchully Pharm. Co. Ltd. (Korea). The mutant (MuT) RNA probes as negative controls were substituted into AGCA from AUUUA sequences of UPA and UPAR ARE-WT. Cytoplasmic extracts were prepared from Res treated U87MG cells using the NEPER Nuclear and Cytoplasmic Extraction Reagent (Thermo Pierce Biotechnology Scientific, USA). RNA EMSA was performed using the LightShift ${ }^{\mathrm{TM}}$ Chemiluminescent EMSA Kit.

\section{Methylthiazoltetrazolium (MTT) assay}

U87MG cells were seeded in 96-well plates at a density of $3 \times$ $10^{3}$ cells/well. After the designated treatments, $100 \mu \mathrm{l}$ of MTT solution $(2 \mathrm{mg} / \mathrm{ml})$ were added to each well, and the cells were incubated at $37^{\circ} \mathrm{C}$ for an additional $2 \mathrm{~h}$. Dimethyl sulfoxide (DMSO) was added and mixed thoroughly, and the absorbance was measured at $570 \mathrm{~nm}$ using an Infinite M200 Pro (Tecan).

\section{TdT-mediated dUTP-biotin nick end labeling (TUNEL)} assay

Cells were seeded on pre-coated coverslips (Nalge Nunc International, USA). Following treatment, the cells were fixed in $4 \%$ paraformaldehyde in phosphate-buffered saline (PBS) for 10 min followed by permeabilization with $0.1 \%$ Triton X-100 in PBS for $5 \mathrm{~min}$. After three washes with PBS, the specimens were labeled using an In Situ Cell Death Detection Kit (TMR red) (Roche Applied Science). Fluorescent images were observed using a DSU fluorescence microscope (BX 51; Olympus, Japan) coupled to a CCD camera (DP72, Olympus).

\section{Transfection with small interfering RNAs (siRNAs)}

The siRNAs against human TTP (sc-36761) and control siRNA (scrambled siRNA [scRNA], (sc-37007) were purchased from Santa Cruz Biotechnology (USA). U87MG cells were transfected with TTP-siRNA using Lipofectamine ${ }^{\mathrm{TM}}$ RNAiMAX (Invitrogen). At $24 \mathrm{~h}$ after transfection, U87MG cells were treated with Res.

\section{Statistical analysis}

Data are expressed as the mean \pm SEM. Statistical significance was determined using Student's $t$-test (Prism; GraphPad Software, Inc., USA). $P$-values $<0.05$ were considered statistically significant (e.g., ${ }^{*} P<0.05,{ }^{* \star} P<0.01$, and ${ }^{* * *} P<0.001$ ). 

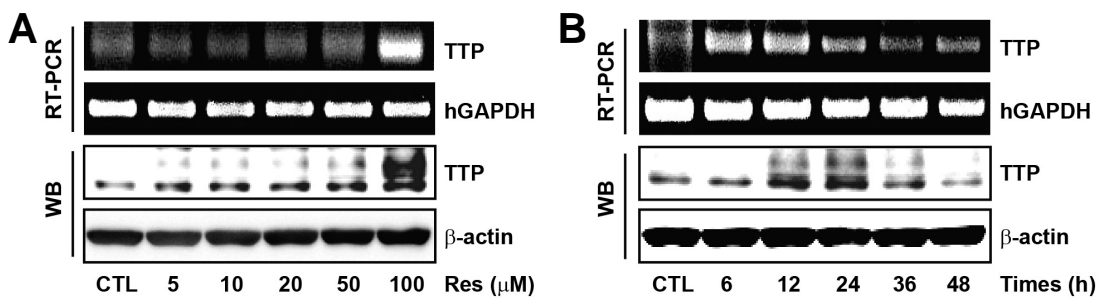

Fig. 1. Res induces endogenous expression of TTP in U87MG human glioma cells. (A) U87MG cells were treated with Res at the indicated concentrations for $12 \mathrm{~h}$ (mRNA) and $24 \mathrm{~h}$ (protein), respectively. Expression of TTP was determined by RT-PCR and western blotting. hGAPDH and $\beta$-actin were used as loading controls. (B) U87MG cells were treated with $100 \mu \mathrm{M}$ Res for the indicated times.

\section{RESULTS}

Res induces endogenous expression of TTP in U87MG human glioma cells

To investigate the effects of Res on the endogenous expression of TTP in U87MG human glioma cells, TTP mRNA and protein expression were measured by RT-PCR and Western blotting, respectively. Cells were treated with dose- and time-dependent Res treatments. Both TTP mRNA and protein expression were significantly increased by the treatment with $100 \mu \mathrm{M}$ of Res (Fig. $1 \mathrm{~A})$. Furthermore, Res treatment $(100 \mu \mathrm{M})$ maximally increased the expression of TTP mRNA and protein at $12 \mathrm{~h}$ and $24 \mathrm{~h}$, respectively (Fig. 1B). These results show that Res increased the expression of endogenous TTP in U87MG cells.

\section{Res-induced TTP leads to the decay of UPA and UPAR} mRNAs

A previous study reported that TTP mediated the stability of UPA and UPAR transcripts associated with the invasion and tumor growth in human breast cancer cells (Al-Souhibani et al., 2010). To test the effects of Res-induced TTP on UPA and UPAR expression, the expression of UPA and UPAR mRNAs and protein were determined by RT-PCR and Western blotting, respectively. Res treatment increased the expression of TTP, and both UPA and UPAR expression were inversely diminished by Res treatment (Fig. 2A). These results show that increased expression of TTP by Res inhibited UPA and UPAR expression in U87MG cells.

To determine whether down-regulation of UPA and UPAR ex- pression by Res-induced TTP was regulated through the decay of their mRNAs, the half-life of these mRNAs was assessed by Q-PCR in U87MG cells. In U87MG cells treated with DMSO (U87MG/CTL), both UPA and UPAR mRNAs were stable for $2 \mathrm{~h}$ and $3 \mathrm{~h}$ after actinomycin $\mathrm{D}$ treatment, respectively. In contrast, in U87MG cells treated with Res (U87MG/Res), the half-life of these mRNAs decreased to less than $2 \mathrm{~h}$ and $3 \mathrm{~h}$, respectively (Fig. 2B). These results indicate that Res-induced TTP led to the decay of UPA and UPAR mRNAs.

\section{The UPA and UPAR AREs are essential for the inhibitory} effect of Res-induced TTP

To determine whether Res-induced TTP mediated destabilization of UPA and UPAR mRNAs in association with $3^{\prime}$ UTR of their mRNAs, U87MG cells were co-transfected with the psiCHECK2 luciferase expression vector containing uPA or UPAR 3' UTR fragments. After being transfected with pcDNA6/V5-TTP, U87MG cells treated with Res significantly inhibited the luciferase activity of UPA and UPAR (Figs. 3B and $3 C$ ). These results suggest that the $3^{\prime}$ UTR of UPA and UPAR mRNAs might be essential for destabilization of their mRNAs through the inhibitory activity of Res-induced TTP.

\section{Res-induced TTP binds to UPA and UPAR AREs}

To investigate whether Res-induced TTP directly binds to the ARE regions containing the 3' UTR of UPA and UPAR mRNAs, RNA EMSA was conducted using biotinylated RNA probes containing the WT or MuT AREs of UPA and UPAR. The RNA probes used in RNA EMSA were the same as those used in the
$\boldsymbol{A}$

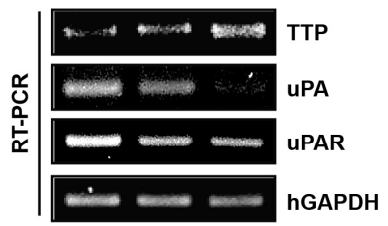

B

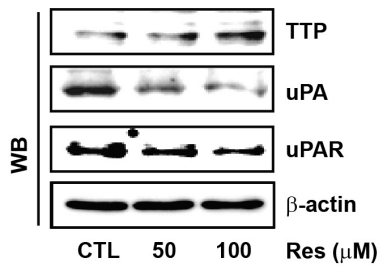

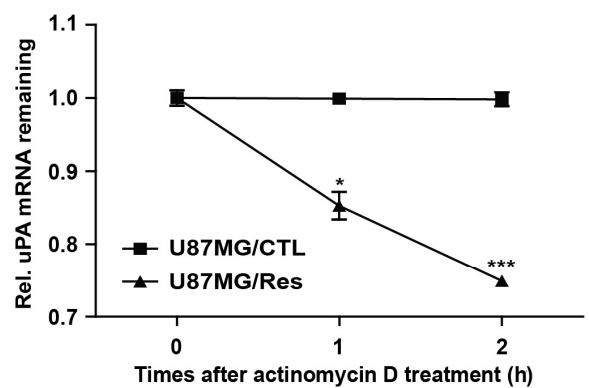

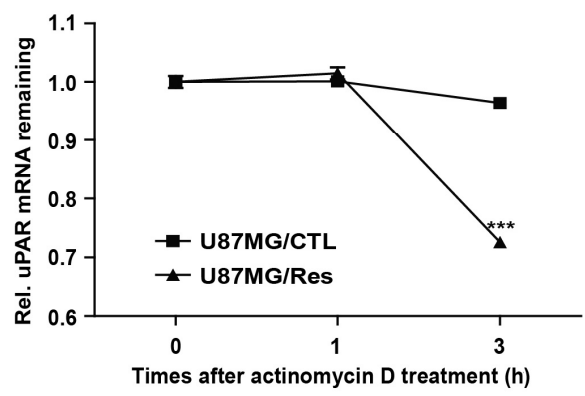

Fig. 2. Res-induced TTP leads to the decay of UPA and UPAR mRNAs. (A) U87MG cells were treated at the indicated concentrations. The expression levels of TTP, UPA, and UPAR were measured by RT-PCR and Western blotting. hGAPDH and $\beta$-actin were used as loading controls. (B) U87MG cells were treated with $100 \mu \mathrm{M}$ Res for $12 \mathrm{~h}$. The expression of UPA and UPAR mRNAs were determined by Q-PCR at the indicated times after the addition of $5 \mu \mathrm{g} / \mathrm{ml}$ actinomy$\operatorname{cin} \mathrm{D}$. The results are expressed as the means \pm SEM. of three independent experiments $\left({ }^{*} P<\right.$ $\left.0.05,{ }^{* \star \star} P<0.001\right)$. 
$\boldsymbol{A}$

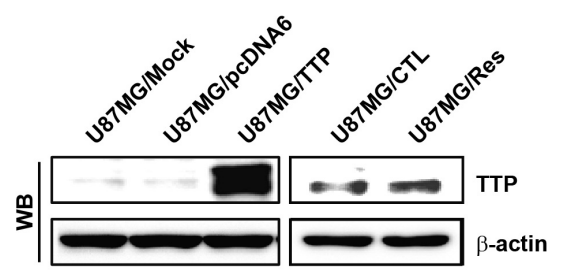

B

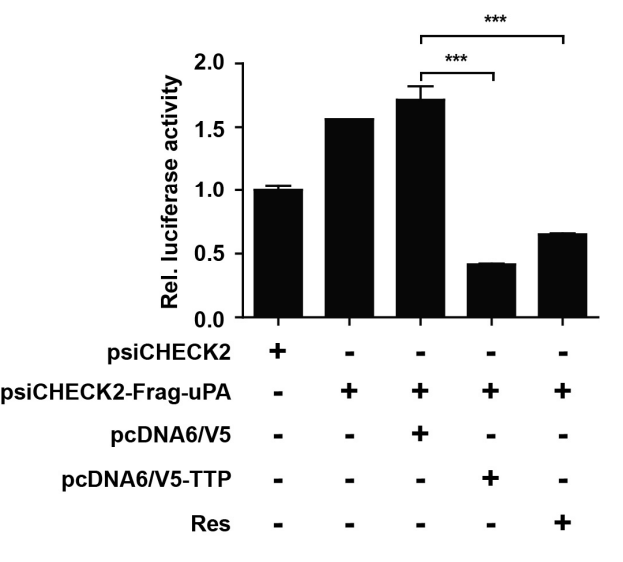

C

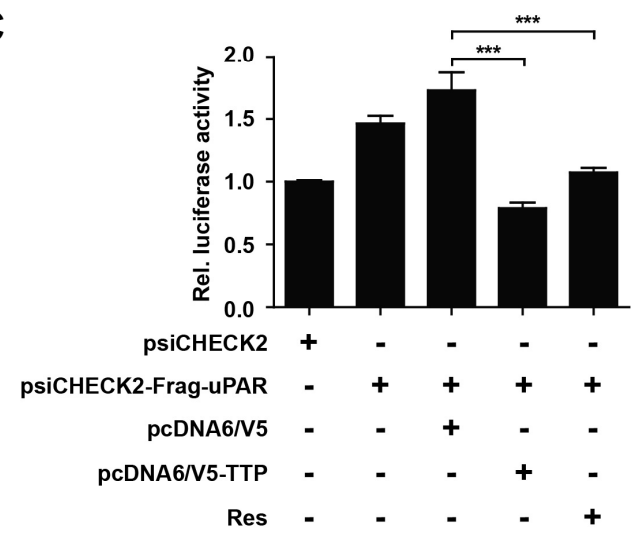

luciferase assay. The MuT RNA probes serving as negative controls were substituted into AGCA from AUUUA sequences of UPA and UPAR ARE-WT. Cytoplasmic extracts were prepared from U87MG cells treated with Res and incubated with biotinylated RNA probes containing the WT or MuT AREs of UPA and UPAR 3' UTR. When RNA EMSA was performed using the UPA-ARE-WT and UPAR-ARE-WT probes, a dominant RNA-protein complex was observed. However, both uPA-AREMuT and UPAR-ARE-MuT prevented the formation of this complex. Complex formation was decreased with an anti-TTP antibody (Ab) (Fig. 4). Together, these results indicate that Resinduced TTP controlled the expression of UPA and UPAR mRNAs in an AREs-dependent manner.

\section{Res induces glioma cell apoptosis}

To determine whether the expression of Res-induced TTP affected human glioma cell growth, glioma cell growth was measured for $72 \mathrm{~h}$ using the MTT assay. Res treatment (5-200 $\mu \mathrm{M})$ was used to treat U87MG cells, and showed growth inhibition using a concentration of $100 \mu \mathrm{M}$ at $24 \mathrm{~h}$. At $72 \mathrm{~h}$ after Res treatment, Res suppressed cell growth at 20-200 $\mu \mathrm{M}$ in a dosedependent manner (Fig. 5A). Res treatment of NHFB cells obtained the same results at $72 \mathrm{~h}$. However, Res treatment of NHFB cells for $24 \mathrm{~h}$ and $48 \mathrm{~h}$ inhibited cell growth at a concentration of $200 \mu \mathrm{M}$ (Supplementary Fig. S1). Taken together, these results suggest that increased expression of TTP by Res inhibited U87MG cell growth.

Previous studies reported that down-regulation of UPA and UPAR activated caspase-mediated apoptosis and inhibited the PI3K/AKT pathway in human glioma cells (Gondi et al., 2007). Figure 2A shows that the increased expression of TTP by Res inhibited UPA and UPAR expression in U87MG cells. TUNEL assay was used to identify whether the expression of Resinduced TTP affected glioma cell apoptosis in the U87MG/Res or U87MG/CTL cells. In U87MG/Res cells, TUNEL-positive cells increased approximately $50 \%$ compared with the U87MG/CTL cells (Figs. 5B and 5C). In addition, the Res treatment raised cleaved caspase-3 expression through upregulation of TTP expression in U87MG cells (Fig. 5D). These results show that the expression of Res potentiated apoptosis in the human glioma cells.

Fig. 3. UPA and uPAR AREs are essential for the inhibitory effect of Res-induced TTP. (A) U87MG cells were transfected with pcDNA6/5-TTP (U87MG/TTP) or pcDNA6/V5 vector (U87MG/pcDNA6). Then, U87MG cells were treated with $100 \mu \mathrm{M}$ Res for $24 \mathrm{~h}$. The expression of TTP was determined by western blotting. $\beta$-actin was used as loading control. $(B, C)$ For the luciferase assay, U87MG cells were co-transfected with pcDNA6/V5-TTP and $0.3 \mu \mathrm{g}$ psiCHECK2 luciferase reporter construct containing various fragments derived from uPA and uPAR mRNA $3^{\prime}$ UTR. After transfection, U87MG cells were treated with $100 \mu \mathrm{M}$ Res for $24 \mathrm{~h}$. Cells were harvested, and luciferase activity was normalized to firefly activity. The results are expressed as the mean \pm SEM. of three independent experiments $\left({ }^{* \star *} P<0.001\right)$.

$\boldsymbol{A}$
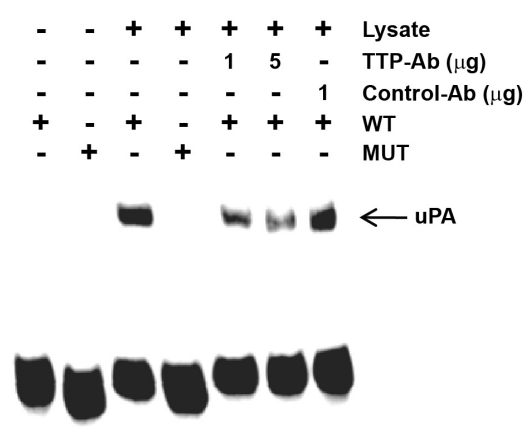

B
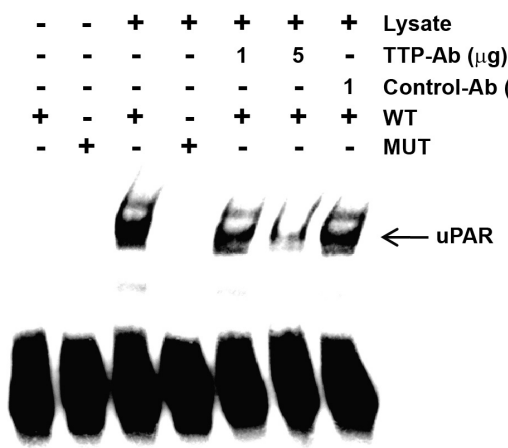

Fig. 4. Res-induced TTP binds to UPA and UPAR AREs. (A, B) RNA EMSA was performed by mixing cytoplasmic g) extract containing $1 \mu \mathrm{g}$ of total protein from Res-treated U87MG cells with 20 fmol of biotinylated UPA and UPAR WT; AUUUA- or MUT; -AGCA- probes. TTP$A b$ or control $A b$ was added to the reaction mixtures. The binding reaction samples were then separated by electrophoresis on a $5 \%$ polyacrylamide gel under nondenaturing conditions. Arrows indicate the position of the RNA-protein complex.

WT : -UAUUUAU

MUT : -UAGCAU- 
$\boldsymbol{A}$

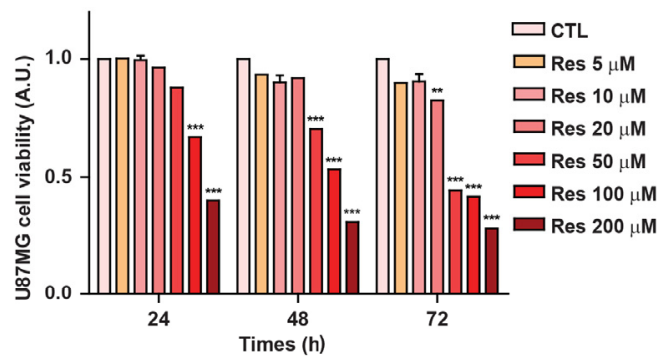

B

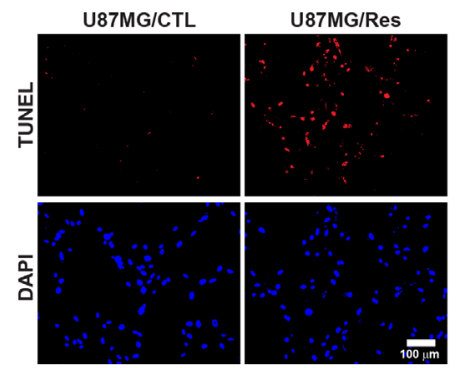

C

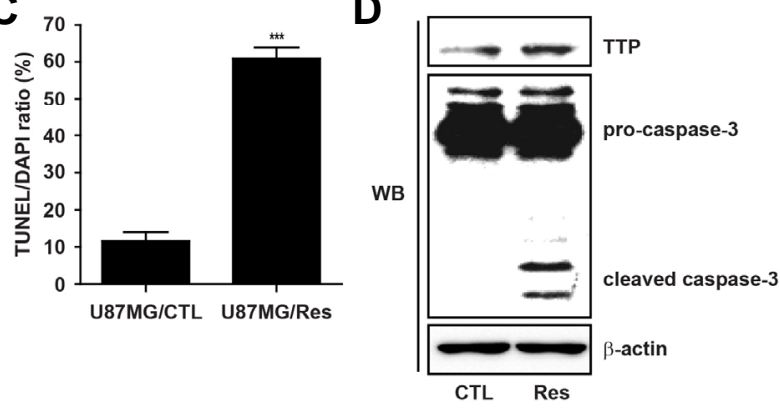

Fig. 5. Res induces glioma cell apoptosis. (A) U87MG cells were treated with Res at the indicated concentrations for $72 \mathrm{~h}$. The MTT assay was used to determine the inhibitory effect of Res-induced TTP on cell growth. Cell survival relative to that of the control (\%) is expressed as the mean \pm SEM. of eight independent experiments ( $\left.{ }^{\star *} P<0.01,{ }^{* \star *} P<0.001\right)$. (B) U87MG cells were treated with 100 $\mu \mathrm{M}$ Res for $24 \mathrm{~h}$. The TUNEL assay was used to determine the effects of Res-induced TTP on glioma cell apoptosis. DAPI was used as a counter-stain. Photographs were taken at a magnification of 100X. (C) Quantification of TUNEL-positive cells was performed using the ratio of TUNEL and DAPI staining. Data are expressed as the mean \pm SEM. of three independent experiments ${ }^{* * *} P<$ 0.0001). (D) Expression levels of TTP and cleaved caspase-3 were determined by Western blotting. The $\beta$-actin was used as loading control.

\section{TTP mediates the apoptotic effect of Res in glioma cells}

To determine whether Res could mediate the apoptotic effects of TTP in glioma cells, we investigated the effects of TTP knock down on Res-induced glioma cell apoptosis. After Res treatment, treatment with siRNA against TTP (TTP-siRNA) significantly inhibited the expression of TTP in U87MG cells (Fig. 6A). Importantly, TTP-siRNA but not scRNA decreased the inhibitory effect of Res on the growth of U87MG cells (Fig. 6B). We also found that TTP-siRNA did not affect Res-induced apoptosis in U87MG cells. Using TTP-siRNA, Res-induced TUNEL-positive cells decreased by approximately $60 \%$ compared with scRNA
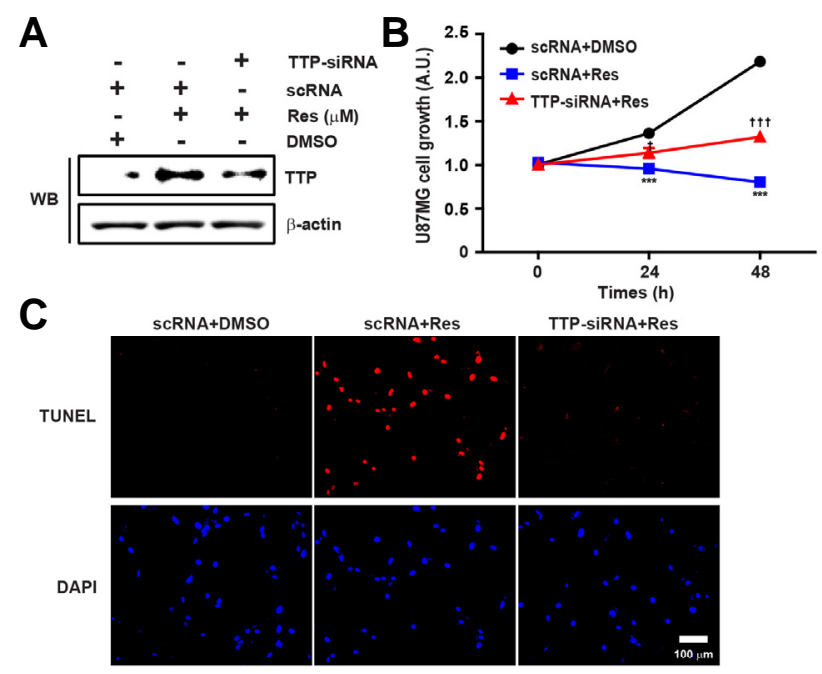

D

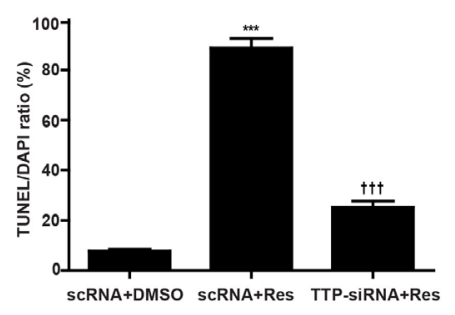

Fig. 6. TTP mediates the apoptotic effect of Res on glioma cells. (A) U87MG cells were transfected with TTP-specific siRNA (TTPsiRNA) or scRNA. After treatment with Res for $24 \mathrm{~h}$, the expression of TTP was measured by Western blotting. The $\beta$-actin was used as loading control. (B) Cell growth was assessed using the MTT assay. Cell survival relative to that of the control (\%) is expressed as the mean \pm SEM. of eight independent experiments. ${ }^{* \star \star} P<0.001$, (vs. scRNA+Res), ${ }^{\dagger} P<0.05,{ }^{\mathrm{tt}} P<0.001$ (vs. TTP-siRNA+Res). (C) The TUNEL assay was used to determine the effects of Resinduced TTP on glioma cell apoptosis. DAPI was used as a counter stain. Photographs were taken at a magnification of 100X. (D) Quantification of TUNEL-positive cells was performed using the ratio of TUNEL and DAPI staining. Data are expressed as the mean \pm SEM. of three independent experiments. ${ }^{* * *} P<0.001$ (vs. scRNA+Res), ${ }^{t+t} P<0.001$ (vs. TTP-siRNA+Res).

(Figs. 6C and 6D). These results suggest that TTP is required for Res-induced apoptosis of U87MG cells.

\section{DISCUSSION}

Despite low expression of TTP in various human cancers (Brennan et al., 2009), the mechanism responsible for this decreased TTP expression is not fully understood. In the present study, we showed that Res, a naturally occurring compound, inhibited cell growth and activated apoptosis through the upregulation of endogenous TTP expression in the human glioma cells.

Previous studies have reported that Res suppressed the invasion, angiogenesis and tumor growth of gliomas (Jiang et al., 2005; Ryu et al., 2011; Tseng et al., 2004). Most importantly, Res was quickly converted into hydrophilic conjugates, that promoted entry into the bloodstream and allowed passage 
through the blood-brain barrier and subsequent absorption into the brain tissue (Wang et al., 2002). These results suggested that Res might be potentially valuable in the treatment of brain tumors (Castino et al., 2011).

The present study shows that Res treatment increased the expression of endogenous TTP in the U87MG human glioma cells (Figs. 1A and 1B). Currently, one mechanism, which explains the lack of TTP in cancers, has been transcriptional silencing as a result of the hypermethylation of CpG islands extant in the TTP promoter (Young et al., 2009). In many disease processes such as cancer, gene promoter $\mathrm{CpG}$ islands are abnormally hypermethylated as a result of a modification of the DNA methyltransferase (DNMT) expression and activity, which results in transcriptional gene silencing (Meeran et al., 2010). Several bioactive dietary components have great potential in altering DNA methylation by altering DNMT levels during cancer prevention and therapy (Li and Tollefsbol, 2010). In previous studies, Res has been shown to have DNMT inhibitory activity. Res interrupted epigenetic silencing of breast cancer-1 (BRCA1 ) induced by the aromatic hydrocarbon receptor (AHR) in the MCF-7 human breast cancer cells (Papoutsis et al., 2010; Stefanska et al., 2010). These results suggested that Res treatment increased the expression of endogenous TTP by blocking the epigenetic silencing of TTP by DNMT inhibitory activity. TTP regulated the decay of UPA and UPAR transcripts associated with the invasion and tumor growth in human breast cancer cells (Al-Souhibani et al., 2010). The present study shows that the increased expression of TTP by Res inhibited the expression of UPA and UPAR mRNAs and proteins (Fig. 2A). Furthermore, the expression of Res-induced TTP promoted the decay of uPA and UPAR mRNAs (Fig. 2B). Posttranscriptional regulation of UPA and UPAR mRNA expression was facilitated by the existence of AREs in the 3'UTR of these mRNAs (Nanbu et al., 1994; Roldan et al., 1990). Specifically, Res-induced TTP directly bound to the ARE regions containing the $3^{\prime}$ UTR of UPA and UPAR mRNAs (Figs. 4A and $4 B$ ). Overall, the results of the present study show that TTP is a regulator of UPA and UPAR mRNA turnover.

The down-regulation of UPA and UPAR using the antisense adenovirus-mediated constructs in SNB19 glioma cells resulted in decreased migration and invasion, and retarded intracranial tumor growth in nude mice (Gondi et al., 2003). The expression of Res-induced TTP in U87MG cells (Fig. 5A) inhibited cell growth compared with the NHFB cells (Supplementary Fig. S1). The down-regulation of UPA and UPAR expression lead to the dephosphorylation of p-Akt, the activation of caspase-8, and a collapse in the mitochondrial membrane potential as revealed by the release of cytochrome $\mathrm{c}$ into the enclosing cytoplasm (Gondi et al., 2007). Together, the results showed that the increased expression of TTP by Res inhibited cell growth through the down-regulation of UPA and UPAR expression in the U87MG cells.

The present study also demonstrated that the expression of Res-potentiated TTP induced apoptosis in the human glioma cells (Figs. 5B-5D). Importantly, the inhibition of TTP by siRNA attenuated the effects of Res on both cell growth (Fig. 6B) and apoptosis (Figs. 6C and 6D). These results indicate that TTP mediated the apoptotic effect of Res on human glioma cells. In many previous studies, TTP has multiple cellular functions, including roles in cell survival, proliferation, differentiation, apoptosis and tumorigenesis. TTP mediated cell-cycle arrest and cell death through regulation of p21/CIP1/WAF-1 (Al-Haj et al., 2012). TTP overexpression also enhances the decay of Pim-1 mRNA by binding to the AU-rich element in its 3'-UTR (Kim et al., 2012). Therefore, TTP directly regulated cell death through decay of p21 mRNA. Furthermore, TTP indirectly regulated cell death through decay of pim1 mRNA. In particular, TTP induced an apoptotic program of cell death in human glioma cells (Suswam et al., 2008). In addition to Res, the other agents such as doxycycline (DOX) also induced the expression of TTP in human cancer cells (Lee et al., 2013). However, it is not known whether TTP induction by other agents involved the same mechanisms therefore further studies are needed to characterize the mechanism mediating TTP expression by diverse agents, including Res.

In summary, the present study demonstrated that Res increased TTP expression in the human glioma cells. The expression of Res-induced TTP were determined to destabilized UPA and UPAR mRNAs by the direct binding to the ARE regions containing the $3^{\prime}$ UTR regions of their mRNAs. Furthermore, the increased expression of TTP by Res suppressed cell growth and induced apoptosis in the human glioma cells. These findings suggest that by regulating TTP expression, several bioactive dietary compounds such as Res can be used as novel anti-cancer agents for the treatment of malignant gliomas.

Note: Supplementary information is available on the Molecules and Cells website (www.molcells.org).

\section{ACKNOWLEDGMENTS}

This work was supported by the National Research Foundation of Korea (NRF) and by a grant funded by the Korean Government (MEST) (No. 2013R1A2A2A01068964).

\section{REFERENCES}

Ahmad, N., Adhami, V.M., Afaq, F., Feyes, D.K., and Mukhtar, H. (2001). Resveratrol causes WAF-1/p21-mediated G(1)-phase arrest of cell cycle and induction of apoptosis in human epidermoid carcinoma A431 cells. Clin. Cancer Res. 7, 14661473.

Al-Haj, L., Blackshear, P.J., and Khabar, K.S. (2012). Regulation of p21/CIP1/WAF-1 mediated cell-cycle arrest by RNase $L$ and tristetraprolin, and involvement of AU-rich elements. Nucleic Acids Res. 40, 7739-7752.

Al-Souhibani, N., Al-Ahmadi, W., Hesketh, J.E., Blackshear, P.J. and Khabar, K.S. (2010). The RNA-binding zinc-finger protein tristetraprolin regulates AU-rich mRNAs involved in breast cancer-related processes. Oncogene 29, 4205-4215.

Baou, M., Jewell, A., and Murphy, J.J. (2009). TIS11 family proteins and their roles in posttranscriptional gene regulation. J. Biomed. Biotechnol. 2009, 634520.

Boocock, D.J., Faust, G.E., Patel, K.R., Schinas, A.M., Brown, V.A., Ducharme, M.P., Booth, T.D., Crowell, J.A., Perloff, M., Gescher, A.J., et al. (2007). Phase I dose escalation pharmacokinetic study in healthy volunteers of resveratrol, a potential cancer chemopreventive agent. Cancer Epidemiol. Biomarkers Prev. 16, 1246-1252.

Brennan, S.E., Kuwano, Y., Alkharouf, N., Blackshear, P.J., Gorospe M., and Wilson, G.M. (2009). The mRNA-destabilizing protein tristetraprolin is suppressed in many cancers, altering tumorigenic phenotypes and patient prognosis. Cancer Res. 69, 5168-5176.

Castino, R., Pucer, A., Veneroni, R., Morani, F., Peracchio, C., Lah, T.T., and Isidoro, C. (2011). Resveratrol reduces the invasive growth and promotes the acquisition of a long-lasting differentiated phenotype in human glioblastoma cells. J. Agric. Food Chem. 59, 4264-4272.

Cottart, C.H., Nivet-Antoine, V., Laguillier-Morizot, C., and Beaudeux, J.L. (2010). Resveratrol bioavailability and toxicity in humans. Mol. Nutr. Food Res. 54, 7-16.

Cucciolla, V., Borriello, A., Oliva, A., Galletti, P., Zappia, V., and Della Ragione, F. (2007). Resveratrol: from basic science to the clinic. Cell Cycle 6, 2495-2510.

Dorai, T., and Aggarwal, B.B. (2004). Role of chemopreventive 
agents in cancer therapy. Cancer Lett. 215, 129-140.

Fremont, L. (2000). Biological effects of resveratrol. Life Sci. 66, 663-673.

Gondi, C.S., Lakka, S.S., Yanamandra, N., Siddique, K., Dinh, D.H., Olivero, W.C., Gujrati, M., and Rao, J.S. (2003). Expression of antisense UPAR and antisense UPA from a bicistronic adenoviral construct inhibits glioma cell invasion, tumor growth, and angiogenesis. Oncogene 22, 5967-5975.

Gondi, C.S., Kandhukuri, N., Dinh, D.H., Gujrati, M., and Rao, J.S. (2007). Down-regulation of UPAR and UPA activates caspasemediated apoptosis and inhibits the PI3K/AKT pathway. Int. J. Oncol. 31, 19-27.

Jang, M., Cai, L., Udeani, G.O., Slowing, K.V., Thomas, C.F., Beecher, C.W., Fong, H.H., Farnsworth, N.R., Kinghorn, A.D., Mehta, R.G., et al. (1997). Cancer chemopreventive activity of resveratrol, a natural product derived from grapes. Science 275 , 218-220.

Jiang, H., Zhang, L., Kuo, J., Kuo, K., Gautam, S.C., Groc, L., Rodriguez, A.I., Koubi, D., Hunter, T.J., Corcoran, G.B., et al. (2005). Resveratrol-induced apoptotic death in human U251 glioma cells. Mol. Cancer Ther. 4, 554-561.

Kim, H.K., Kim, C.W., Vo, M.T., Lee, H.H., Lee, J.Y., Yoon, N.A., Lee, C.Y., Moon, C.H., Min, Y.J., Park, J.W., et al. (2012). Expression of proviral integration site for Moloney murine leukemia virus 1 (Pim-1) is post-transcriptionally regulated by tristetraprolin in cancer cells. J. Biol. Chem. 287, 28770-28778.

Lee, H.H., Vo, M.T., Kim, H.J., Lee, U.H., Kim, C.W., Kim, H.K., Ko, M.S., Lee, W.H., Cha, S.J., Min, Y.J., et al. (2010). Stability of the LATS2 tumor suppressor gene is regulated by tristetraprolin. J. Biol. Chem. 285, 17329-17337.

Lee, J.Y., Kim, H.J., Yoon, N.A., Lee, W.H., Min, Y.J., Ko, B.K., Lee, B.J., Lee, A., Cha, H.J., Cho, W.J., et al. (2013). Tumor suppressor p53 plays a key role in induction of both tristetraprolin and let-7 in human cancer cells. Nucleic Acids Res. 41, 56145625.

$\mathrm{Li}$, Y., and Tollefsbol, T.O. (2010). Impact on DNA methylation in cancer prevention and therapy by bioactive dietary components. Curr. Med. Chem. 17, 2141-2151.

Meeran, S.M., Ahmed, A., and Tollefsbol, T.O. (2010). Epigenetic targets of bioactive dietary components for cancer prevention and therapy. Clin. Epigenetics 1, 101-116.

Nakada, M., Nakada, S., Demuth, T., Tran, N.L., Hoelzinger, D.B., and Berens, M.E. (2007). Molecular targets of glioma invasion. Cell Mol. Life Sci. 64, 458-478.

Nanbu, R., Menoud, P.A., and Nagamine, Y. (1994). Multiple instability-regulating sites in the $3^{\prime}$ untranslated region of the urokinase-type plasminogen activator mRNA. Mol. Cell Biol. 14, 4920-4928.

Papoutsis, A.J., Lamore, S.D., Wondrak, G.T., Selmin, O.I., and Romagnolo, D.F. (2010). Resveratrol prevents epigenetic silencing of BRCA-1 by the aromatic hydrocarbon receptor in human breast cancer cells. J. Nutr. 140, 1607-1614.

Roldan, A.L., Cubellis, M.V., Masucci, M.T., Behrendt, N., Lund, L.R.,
Dano, K., Appella, E., and Blasi, F. (1990). Cloning and expression of the receptor for human urokinase plasminogen activator, a central molecule in cell surface, plasmin dependent proteolysis. EMBO J. 9, 467-474.

Ryu, J., Ku, B.M., Lee, Y.K., Jeong, J.Y., Kang, S., Choi, J., Yang, Y., Lee, D.H., Roh, G.S., Kim, H.J., et al. (2011). Resveratrol reduces TNF-alpha-induced U373MG human glioma cell invasion through regulating NF-kappaB activation and uPA/uPAR expression. Anticancer Res. 31, 4223-4230.

Ryu, J., Yoon, N.A., Lee, Y.K., Jeong, J.Y., Kang, S., Seong, H., Choi, J., Park, N., Kim, N., Cho, W.J., et al. (2015). Tristetraprolin inhibits the growth of human glioma cells through downregulation of urokinase plasminogen activator/urokinase plasminogen activator receptor mRNAs. Mol. Cells 38, 156-162.

Stefanska, B., Rudnicka, K., Bednarek, A., and FabianowskaMajewska, K. (2010). Hypomethylation and induction of retinoic acid receptor beta 2 by concurrent action of adenosine analogues and natural compounds in breast cancer cells. Eur. J. Pharmacol. 638, 47-53.

Stupp, R., Hegi, M.E., Gilbert, M.R., and Chakravarti, A. (2007). Chemoradiotherapy in malignant glioma: standard of care and future directions. J. Clin. Oncol. 25, 4127-4136.

Suswam, E., Li, Y., Zhang, X., Gillespie, G.Y., Li, X., Shacka, J.J., Lu, L., Zheng, L., and King, P.H. (2008). Tristetraprolin downregulates interleukin-8 and vascular endothelial growth factor in malignant glioma cells. Cancer Res. 68, 674-682.

Tinhofer, I., Bernhard, D., Senfter, M., Anether, G., Loeffler, M. Kroemer, G., Kofler, R., Csordas, A., and Greil, R. (2001). Resveratrol, a tumor-suppressive compound from grapes, induces apoptosis via a novel mitochondrial pathway controlled by Bcl-2. FASEB J. 15, 1613-1615.

Trincheri, N.F., Nicotra, G., Follo, C., Castino, R., and Isidoro, C. (2007). Resveratrol induces cell death in colorectal cancer cells by a novel pathway involving lysosomal cathepsin D. Carcinogenesis 28, 922-931.

Tseng, S.H., Lin, S.M., Chen, J.C., Su, Y.H., Huang, H.Y., Chen, C.K., Lin, P.Y., and Chen, Y. (2004). Resveratrol suppresses the angiogenesis and tumor growth of gliomas in rats. Clin. Cancer Res. 10, 2190-2202.

Villano, J.L., Seery, T.E., and Bressler, L.R. (2009). Temozolomide in malignant gliomas: current use and future targets. Cancer Chemother Pharmacol. 64, 647-655.

Wang, Q., Xu, J., Rottinghaus, G.E., Simonyi, A., Lubahn, D., Sun G.Y., and Sun, A.Y. (2002). Resveratrol protects against global cerebral ischemic injury in gerbils. Brain Res. 958, 439-447.

Weng, C.J., Wu, C.F., Huang, H.W., Wu, C.H., Ho, C.T., and Yen G.C. (2010). Evaluation of anti-invasion effect of resveratrol and related methoxy analogues on human hepatocarcinoma cells. J. Agric. Food Chem. 58, 2886-2894.

Young, L.E., Sanduja, S., Bemis-Standoli, K., Pena, E.A., Price, R.L., and Dixon, D.A. (2009). The mRNA binding proteins HuR and tristetraprolin regulate cyclooxygenase 2 expression during colon carcinogenesis. Gastroenterology 136, 1669-1679. 